\title{
Fatores Relacionados aos Conhecimentos em Nutrição de Puérperas Acompanhadas em Unidades de Saúde da Família
}

\author{
Lígia Rejane Siqueira Garcia ${ }^{1}$, Caroline Sousa Cabral ${ }^{2}$ \\ Karolinny Xavier Alves ${ }^{3}$, Tatiana da Costa Vicente ${ }^{4}$
}

\begin{abstract}
RESUMO
Este estudo objetivou avaliar os fatores relacionados aos conhecimentos em nutrição das puérperas no pós-parto imediato. Trata-se de um estudo transversal, com amostra definida por conveniência, totalizando cem mulheres que foram entrevistadas utilizando questionário de perguntas abertas e fechadas. Os conhecimentos em nutrição foram categorizados por níveis, com base na quantidade de respostas corretas para as 17 perguntas realizadas sobre micronutrientes de importância para o período gestacional (ácido fólico, ferro e vitamina A). Nos resultados verificamos que $77 \%$ receberam orientações nutricionais. No que diz respeito à variável anos de estudo, percebe-se que puérperas que tinham mais de dez anos de estudos apresentaram maior chance de ter conhecimentos sobre o ácido fólico e ferro. Já no que se refere ao recebimento de orientações alimentares, puérperas que vivenciaram esta prática durante o pré-natal apresentaram maior chance de conhecer informações sobre o ácido fólico e a vitamina A. Os conhecimentos em nutrição de puérperas acompanhadas pelo pré-natal em Unidades de Saúde da Família são influenciados por múltiplos fatores, dentre os quais: os anos de estudo, quantidade de consultas pré-natal, participação em palestras educativas, orientações alimentares e nutricionais. Isso que reforça a necessidade de fortalecimento de ações de educação alimentar e nutricional durante o acompanhamento pré-natal para favorecer a saúde materna e infantil.
\end{abstract}

Palavras-chave: Saúde da criança. Promoção da saúde. Cuidado pré-natal. Nutrição em saúde pública.

FACTORS RELATED TO KNOWLEDGE IN NUTRITION OF PUERPERAL WOMEN ACCOMPANIED IN FAMILY HEALTH UNITS

\begin{abstract}
This study aimed to evaluate the factors related to the nutritional knowledge of puerperal women in the immediate postpartum period. This is a cross-sectional study, with a sample defined by convenience, totaling one hundred women who were interviewed using a questionnaire of open and closed questions. The knowledge in nutrition was categorized by levels, based on the number of correct answers for the 17 questions about micronutrients of importance for the gestational period (folic acid, iron and vitamin A). In the results we found that $77 \%$ received nutritional guidance. About the variable years of study, it is perceived that puerperal women who had more than ten years of studies presented a greater knowledge about folic acid and iron. Regarding the receipt of dietary guidelines, puerperal women who experienced this practice during the prenatal period presented a greater chance of knowing information about folic acid and vitamin A. The knowledge on nutrition of puerperal women accompanied by prenatal care in family health units are influenced by multiple factors, among which: the years of study, quantity of prenatal consultations, participation in educational lectures, dietary and nutritional orientations. This reinforces the need to strengthen food and nutrition education actions during prenatal care to promote maternal and child health.
\end{abstract}

Keywords: Child health. Health promotion. Prenatal care. Nutrition in public health.

\footnotetext{
${ }^{1}$ Doutora em Saúde Coletiva pela Universidade Federal do Rio Grande do Norte (UFRN). Docente do curso de Nutrição da FACISA-UFRN. ligiarejane@yahoo.com.br

2 Doutoranda em Nutrição pela Universidade Federal da Paraíba - PB. carolinescabral@hotmail.com

${ }^{3}$ Nutricionista pela Universidade Potiguar - RN. karolinnyalves20@hotmail.com

${ }^{4}$ Nutricionista pela Universidade Potiguar - RN. vicentetati@outlook.com
} 


\section{INTRODUÇÃO}

A gestação é um período em que as necessidades nutricionais se encontram elevadas e, portanto, a alimentação saudável e adequada é essencial para a saúde da mãe e do bebê. As gestantes devem consumir alimentos em quantidades e variedades específicas, considerando as indicações dos guias alimentares e as práticas alimentares culturais, para atingir as necessidades energéticas e nutricionais e as recomendações do ganho de peso (MELERE et al., 2013). A participação de micronutrientes é fundamental para a condução de uma gestação saudável, haja vista que os mesmos estão envolvidos em processos fisiológicos fundamentais para a saúde da gestante e do feto (VITOLO, 2015).

As gestantes brasileiras vêm apresentando dados preocupantes acerca de sua saúde e nutrição. Pesquisas sugerem o crescimento de patologias carenciais, as quais podem ser bastante prejudiciais à saúde materno-infantil. Dentre as patologias com maior prevalência, identifica-se a deficiência de vitamina $A$ e a anemia ferropriva (RAMALHO et al., 2006; BRASIL, 2009b).

A orientação da gestante deve fomentar a adesão a uma alimentação de boa qualidade e saudável que supra as suas necessidades nutricionais, garantindo, assim, o crescimento e o desenvolvimento adequado do feto. É necessário evitar o consumo excessivo de gordura e açúcares, além de evitar o sedentarismo, os quais podem colaborar no ganho de peso excessivo e outros distúrbios metabólicos (CAMARGO et al., 2012).

A atenção nutricional no pré-natal é essencial para a saúde materna e da criança, tendo como objetivo acolher a gestante desde o início da gravidez, assegurando o nascimento de uma criança saudável. O monitoramento contínuo do estado nutricional, durante esse período, é fundamental para ganho de peso ideal durante toda a gestação (BRASIL, 2013). Uma atenção pré-natal e puerperal qualificada e humanizada se dá por meio da incorporação de condutas acolhedoras, facilitando o acesso a serviços de saúde de qualidade, com ações que integrem todos os níveis da atenção: prevenção e promoção da saúde, além de diagnóstico e tratamento adequado dos problemas que ocorrem neste período (BRASIL, 2005).

Neste sentido, a alimentação e a nutrição são requisitos básicos para a promoção e a proteção da saúde, possibilitando, assim, crescimento e desenvolvimento humanos com uma boa qualidade de vida (BRASIL, 2013). As ações de alimentação e nutrição, portanto, representam papel fundamental no contexto da Atenção Básica em Saúde, em especial na Estratégia da Saúde da Família (BRASIL, 2009a).

Mesmo diante das problemáticas identificadas, a estrutura do processo de assistência pré-natal e puerperal ainda é bastante centrada no cuidado médico, permitindo inferir o fortalecimento das ações de cura e tratamento em detrimento das práticas preventivas e de promoção (ROCHA; ANDRADE, 2008).

Em virtude da insuficiência de profissionais nutricionistas inseridos na Atenção Básica, é questionado se o acompanhamento pré-natal está sendo suficiente para trazer conhecimentos em nutrição para a gestante. Os dados na literatura são escassos, respaldando a necessidade da realização de estudos nesta perspectiva. Diante do exposto, este estudo tem como objetivo investigar os fatores relacionados aos conhecimentos em nutrição das puérperas no pós-parto imediato, internadas em maternidade de um município do Nordeste brasileiro.

\section{MÉTODO}

A presente pesquisa caracteriza-se como um estudo observacional, quantitativo, do tipo transversal, cuja unidade amostral são puérperas de uma maternidade localizada no Nordeste brasileiro, previamente acompanhadas pelo pré-natal das Unidades de Saúde da Família do mesmo município.

A amostra do presente estudo foi realizada por conveniência, quando foram pesquisadas todas as puérperas internas na maternidade durante os meses de agosto e setembro de 2015, com uma amostra de cem puérperas.

Para a seleção das participantes foi estabelecido como critérios de inclusão residir no município de Parnamirim/RN, ser cadastrada em Unidades de Saúde da Família do respectivo município e ser puérpera no pós-parto imediato. Como critérios de exclusão destacam-se não querer participar da pesquisa, bem como todos os elementos que não atendam às especificações de inclusão.

O protocolo da pesquisa foi aprovado pelo Comitê de Ética em Pesquisa da Universidade Potiguar do Rio Grande do Norte (UnP), conforme CAAE № 48721315.0.00005296. Para a coleta de dados, as puérperas foram esclarecidas sobre os objetivos, metodologia da pesquisa e confidencialidade dos dados. Aquelas que concordaram em participar da pesquisa assinaram do Termo de Consentimento Livre e Esclarecido e foi aplicado o instrumento da pesquisa por entrevistadores treinados. 
O questionário era composto por 17 perguntas sobre os conhecimentos das puérperas acerca da nutrição, as quais foram categorizadas em níveis de conhecimento com base na quantidade de respostas corretas para as perguntas realizadas. Desta forma, criou-se as seguintes categorias de conhecimento: pouco conhecimento (zero a 5 acertos); conhecimento mediano (6 a 12 acertos) e conhecimento relevante (mais de 13 acertos). Posteriormente, os conhecimentos em nutrição foram categorizados por micronutrientes avaliados. Desta forma, adotou-se que a puérpera possuía conhecimento a respeito do micronutriente avaliado quando ela respondeu positivamente à pergunta "Você conhece o micronutriente ácido fólico/ferro/vitamina A?", e ainda quando diagnosticou corretamente as fontes alimentares dos micronutrientes. Então, criou-se a variável "micronutriente conhecido". Posteriormente, os diferentes fatores intervenientes foram relacionados às categorias de micronutrientes conhecidos.

Utilizou-se o teste qui-quadrado para se verificar a relação dos diferentes fatores sobre esses níveis de conhecimento, bem como em relação às categorias de micronutrientes conhecidos. Adotou-se intervalo de confiança de $95 \%$ e $p \leq 0,05$ para significância estatística.

\section{RESULTADOS}

Foram entrevistadas cem puérperas, com a idade média de 26,3 anos (DP $\pm 7,1$ ) e renda familiar per capita média de $\mathrm{R} \$ 1.103,90$ (DP $\pm 600,90)$. Em relação ao grau de instrução, $96 \%$ das entrevistadas sabiam ler e escrever, com uma média de anos de estudo de 8,6 anos (DP $\pm 4,1$ ). Em relação ao acompanhamento do pré-natal, $98 \%$ das mulheres iniciaram as consultas no terceiro mês de gestação, com uma frequência média de 8 consultas. Durante este acompanhamento, apenas $39 \%$ participaram de palestras educativas e $77 \%$ receberam algum tipo de orientação alimentar. Ao serem questionadas sobre a importância da alimentação durante o período gestacional para a saúde materno-infantil, $86 \%$ delas consideraram importante, embora $8 \%$ tenham apresentado pouco e $46 \%$ mediano nível de conhecimento em nutrição.

Um dado referente ao acompanhamento pré-natal a ser ressaltado é que $16 \%$ das mulheres afirmaram não ter recebido orientação a respeito da importância do aleitamento materno e 3\% não responderam a este questionamento. Outro resultado que chama atenção neste estudo, é que $64 \%$ das puérperas encontrava-se em algum nível de Insegurança Alimentar e Nutricional. A forma mais grave de insegurança alimentar foi encontrada em $16 \%$ das puérperas.

Tabela 1 - Fatores intervenientes no nível de conhecimento em nutrição de puérperas de Parnamirim/RN, 2015

\begin{tabular}{|c|c|c|c|c|c|}
\hline \multirow[t]{2}{*}{ Fatores intervenientes } & \multicolumn{3}{|c|}{ Nível de conhecimento } & \multirow{2}{*}{$\begin{array}{l}\text { Total } \\
\mathrm{N}(\%)\end{array}$} & \multirow[t]{2}{*}{ p-valor } \\
\hline & $\begin{array}{c}\text { Pouco } \\
\mathrm{N}(\%)\end{array}$ & $\begin{array}{c}\text { Mediano } \\
\mathrm{N}(\%)\end{array}$ & $\begin{array}{c}\text { Relevante } \\
\mathrm{N}(\%)\end{array}$ & & \\
\hline Estado de Seg. Alimentar $(n=89)$ & & & & & 0,38 \\
\hline Segurança Alimentar & $3(9,4)$ & $11(34,4)$ & $18(56,2)$ & $32(36)$ & \\
\hline Insegurança Alimentar & $5(8,8)$ & $28(49,1)$ & $24(42,1)$ & $57(64)$ & \\
\hline Anos de Estudo $(n=100)$ & & & & & 0,02 \\
\hline$\leq 5$ anos & $5(18,5)$ & $15(55,6)$ & $7(25,9)$ & $27(27)$ & \\
\hline$>5$ a 10 anos & $1(3,3)$ & $16(53,3)$ & $13(43,3)$ & $30(30)$ & \\
\hline$>10$ anos & $2(4,7)$ & $15(34,9)$ & $26(60,5)$ & $43(43)$ & \\
\hline No de Consultas pré-natal $(n=100)$ & & & & & $<0,01$ \\
\hline$\leq 4$ consultas & $2(14,3)$ & $11(78,6)$ & $1(7,1)$ & $14(14)$ & \\
\hline 5 a 8 consultas & $4(8,7)$ & $22(47,8)$ & $20(43,5)$ & $46(46)$ & \\
\hline Mais de 8 consultas & $2(5)$ & $13(32,5)$ & $25(62,5)$ & $40(40)$ & \\
\hline Início do acompanhamento pré-natal $(n=100)$ & & & & & 0,05 \\
\hline$\leq 3$ meses & $5(6,1)$ & $35(42,7)$ & $42(51,2)$ & $82(82)$ & \\
\hline Após 3 meses & $3(16,7)$ & $11(61,1)$ & $4(22,2)$ & $18(18)$ & \\
\hline Participação em palestras educativas ( $n=97$ ) & & & & & 0,65 \\
\hline Sim & $2(5,1)$ & $18(46,2)$ & $19(48,7)$ & $39(40,2)$ & \\
\hline Não & $6(10,3)$ & $26(44,8)$ & $26(44,8)$ & $58(59,8)$ & \\
\hline Recebimento de orientações alimentares $(n=98)$ & & & & & 0,02 \\
\hline Sim & $4(5,1)$ & $33(42,3)$ & $41(52,6)$ & $78(79,6)$ & \\
\hline Não & $4(20)$ & $11(55)$ & $5(25)$ & $20(20,4)$ & \\
\hline
\end{tabular}


Dados da Tabela 1 demonstram a relação do nível de conhecimento em nutrição das puérperas com diferentes fatores intervenientes. Conforme pode ser observado, as variáveis que apresentaram relação significativa com o melhor nível de conhecimento em nutrição foram: anos de estudo $(p=0,02)$, o maior número de consultas pré-natal $(p<0,01)$, início do pré-natal antes dos 3 meses $(p=0,05)$ e o recebimento de orientações alimentares $(p=0,02)$.

A Tabela 2 demonstra o risco relativo dos diferentes fatores sobre o conhecimento das puérperas no que diz respeito aos micronutrientes. $O$ estado de segurança alimentar não influenciou o conhecimento sobre os nutrientes avaliados. No que se refere à variável anos de estudo, percebe-se que puérperas que tinham mais de dez anos de estudo apresentaram maior risco de ter conhecimentos sobre o ácido fólico e ferro, porém esta variável não influenciou o conhecimento dessas usuárias sobre a vitamina $\mathrm{A}$. Tendo em consideração o acompanhamento pré-natal, quando se avaliou o número de consultas, verificou-se que puérperas que compareceram a mais de 8 consultas apresentaram maior risco de conhecerem o ácido fólico e o ferro, porém nota-se que não houve relação com a vitamina A. Percebeu-se, ainda, que quando o início deste acompanhamento ocorre após os 3 meses, o risco das puérperas conhecerem o ácido fólico diminui em $65 \%$.

Quanto ao recebimento de orientações alimentares, puérperas que vivenciaram esta prática durante o pré-natal apresentaram maior risco de conhecerem informações sobre o ácido fólico e a vitamina A, porém o mesmo não se verificou em relação ao ferro.

\section{DISCUSSÃO}

A gestação e a lactação são momentos biológicos que merecem o máximo de atenção com relação à oferta de micronutrientes como a vitamina $A$, o ferro e o ácido fólico, tendo em vista que a deficiência desses nutrientes pode predispor a uma série de efeitos deletérios para o binômio mãe-filho, com consequente aumento das taxas de morbimortalidade, dentre outros agravos à saúde (SILVA et al., 2007).

Neste estudo fica claro que as puérperas reconhecem a importância da alimentação saudável para o período gestacional. $\mathrm{O}$ acesso a informação limitado

Tabela 2 - Fatores associados aos conhecimentos em micronutrientes específicos dentre puérperas

\begin{tabular}{|c|c|c|c|}
\hline Fatores intervenientes & \multicolumn{3}{|c|}{ Nível de conhecimento } \\
\hline & Pouco & Mediano & Relevante \\
\hline \multicolumn{4}{|l|}{ Estado de Seg. Alimentar $(n=89)$} \\
\hline Insegurança Alimentar & 1 & 1 & 1 \\
\hline Segurança Alimentar & $0,91(0,38-2,17)$ & $0,50(0,21-1,22)$ & $0,47(0,17-1,29)$ \\
\hline \multicolumn{4}{|l|}{ Anos de Estudo $(n=100)$} \\
\hline$\leq 5$ anos & 1 & 1 & 1 \\
\hline$>5$ a 10 anos & $1,9(0,62-5,89)$ & $1,82(0,61-5,44)$ & $1,34(0,37-4,86)$ \\
\hline$>10$ anos & $5,91(2,02-17,27)^{*}$ & $4,92(1,73-13,97)^{*}$ & $1,16(0,34-3,94)$ \\
\hline \multicolumn{4}{|l|}{ № de Consultas pré-natal $(n=100)$} \\
\hline$\leq 4$ consultas & 1 & 1 & 1 \\
\hline Mais de 8 consultas & $5,5(1,3-22,86)^{*}$ & $5,19(1,37-19,73)^{*}$ & $2(0,381-10,51)$ \\
\hline \multicolumn{4}{|l|}{ Início do acompanhamento pré-natal ( $n=100)$} \\
\hline$\leq 3$ meses & 1 & 1 & 1 \\
\hline Após 3 meses & $0,35(0,11-1,07)^{*}$ & $0,58(0,20-1,63)$ & $0,71(0,18-2,73)$ \\
\hline \multicolumn{4}{|l|}{ Participação em palestras educativas ( $n=97$ ) } \\
\hline Não & 1 & 1 & 1 \\
\hline Sim & $1,71(0,75-3,87)$ & $1,13(0,50-2,54)$ & $1,15(0,43-3,06)$ \\
\hline \multicolumn{4}{|l|}{ Recebimento de orientações alimentares ( $n=98)$} \\
\hline Não & 1 & 1 & 1 \\
\hline Sim & $6,55(0,82-52,13)^{*}$ & $1,67(0,61-4,51)$ & $3,69(1,21-11,14)^{*}$ \\
\hline
\end{tabular}

$\mathrm{p} \leq 0,05$ 
durante o acompanhamento do pré-natal por meio de orientações alimentares e palestras educativas, no entanto, é refletivo no conhecimento insuficiente sobre micronutrientes essenciais nesta fase. Destaca-se, ainda, que nem todas as mulheres incluídas neste estudo foram orientadas sobre o aleitamento materno durante o pré-natal.

Um dos fatores de maior impacto na saúde infantil é a nutrição adequada, principalmente pela influência crucial que o estado nutricional desempenha sobre os riscos de morbimortalidade e sobre o crescimento e o desenvolvimento neste ciclo de vida (RIBAS et al., 1999).

Nessa perspectiva, as ações de incentivo ao aleitamento materno devem estar aliadas às atividades de rotina das Unidades de Estratégia Saúde da Família. Dessa forma, o acompanhamento de maneira assídua e no início da gestação deve ser incentivado para favorecer a aquisição de conhecimentos referentes aos cuidados necessários neste ciclo de vida, os quais contribuirão para uma gestação saudável (VARGAS et al., 2016).

Durante o pré-natal também é importante esclarecer à gestante sobre o tempo ideal de aleitamento materno segundo a Organização Mundial de Saúde, dos problemas e dificuldades durante a amamentação, bem como das consequências do desmame precoce, além da importância de alguns micronutrientes necessários para o crescimento do bebê e o tempo certo para oferecer alimentos complementares (BRASIL, 2009b). Nesse sentido, a educação em saúde durante o pré-natal deve levar em consideração que cada mulher é um sujeito único e que carrega consigo sua própria cultura e conhecimentos, traduzidos por meio de suas vivências, medos, dúvidas, crenças e expectativas (LIBERA et al., 2011).

A baixa escolaridade ou instrução materna apresenta-se como um fator de risco para comprometimentos nutricionais, como o retardo no crescimento. Constata-se, ainda, que a mãe com maior conhecimento pode realizar com mais propriedade os cuidados preventivos e curativos ao seu filho (RISSIN et al., 2011).

Neste estudo, as mães que tinham mais instrução e que foram atendidas com uma frequência mínima de 8 consultas, como é preconizado pela Organização Mundial de Saúde, apresentavam mais conhecimento em nutrição quando comparadas às demais.
Outro ponto de destaque neste estudo foi que a maioria das mães encontravam-se em situação de Insegurança Alimentar e Nutricional, que, associada ao conhecimento insuficiente, contribuiu para o desenvolvimento de uma série de agravos à saúde, como deficiência de micronutrientes específicos ou a desnutrição infantil.

Sabe-se que a Insegurança Alimentar e Nutricional está fortemente associada à desnutrição infantil, um agravo à saúde que ainda não foi superado nacionalmente, ocorrendo prevalências importantes em áreas de baixo nível socioeconômico (VIEIRA; SOUZA; CERVATO-MANCUSO, 2010). Um agravante desta relação é que a desnutrição figura entre as principais causas de mortalidade infantil no Brasil (CALDEIRA et al., 2005).

A inserção do profissional nutricionista na Atenção Primária à Saúde torna-se importante com vistas à promoção da saúde em todas as fases da vida, abordando aspectos da alimentação saudável bem como a questão da segurança alimentar. Segundo Geus et al. (2011), essa inserção é justificada pela sua formação acadêmica, que o capacita a realizar o diagnóstico nutricional da população de acordo com ciclos de vida, de maneira a propor orientações dietéticas necessárias e adequadas aos hábitos da unidade familiar, ao meio cultural e levando em conta a disponibilidade de alimentos. O nutricionista está plenamente capacitado para atuar na Atenção Primária, pois a sua ausência confronta-se com o princípio da integralidade das ações de saúde, uma vez que nenhum outro profissional da saúde possui formação para atuar na área de alimentação e nutrição dentro das comunidades e que sua presença viria a contribuir para a promoção da saúde da população.

Considerando o modelo vigente, um avanço na busca para ampliar o escopo das ações e promover a integralidade do cuidado, foi a instituição dos Núcleos de Apoio à Saúde da Família (Nasfs), que contemplam outras especialidades profissionais, como o nutricionista. No município em estudo os nutricionistas estavam inseridos nos Nasfs.

Pádua e Boog (2006) evidenciam que o número elevado de habitantes ainda é um obstáculo aos serviços de saúde, uma vez que as equipes não são suficientes para atender à população devidamente. Nessa perspectiva, as ações de alimentação e nutrição desenvolvidas por estas equipes podem ser insuficientes diante das necessidades da população e condicionadas à definição de prioridades pelo profissional ou pela própria equipe (CERVATO-MANCUSO et al., 2012). 


\section{CONCLUSÕES}

Conforme pode-se perceber nos resultados da presente análise, os conhecimentos em nutrição de puérperas acompanhadas pelo pré-natal são permeados por múltiplos fatores. Dentre eles podemos destacar anos de estudo, quantidade de consultas pré-natal, participação em palestras educativas e orientações alimentares e nutricionais. Diante do exposto, para que se promova uma melhoria dos conhecimentos avaliados são necessárias melhorias no fortalecimento da educação em saúde,para que elas possam adquirir mais conhecimento desde o período da escola, além de ações multisetoriais como práticas de fortalecimento da adesão dessas usuárias.

No intuito de fortalecer os conhecimentos em nutrição entre as puérperas, fazem-se necessárias ações intersetoriais que possam agir não apenas nos determinantes socioculturais do processo saúde-doença, mas também nas práticas em saúde pautadas na integralidade dos sujeitos inseridos neste contexto. Desta forma, contribui-se na promoção da saúde materno-infantil, fortalecendo o processo de cuidado e a autonomia das mães.

\section{REFERÊNCIAS}

BRASIL. Conselho Nacional de Secretários de Saúde. SUS 20 anos. Brasília: CONASS, 2009a.

. Ministério da Saúde. Manual instrutivo das ações de alimentação e nutrição na Rede Cegonha. Brasília: Ministério da Saúde, 2013.

. Ministério da Saúde. Pré-natal e puerpério: atenção qualificada e humanizada - manual técnico. Brasília: Ministério da Saúde, 2005.

. Ministério da Saúde. Saúde da criança: nutrição infantil: aleitamento materno e alimentação complementar. Brasília: Ministério da Saúde, 2009b.

CALDEIRA, A. P. et al. Evolução da mortalidade infantil por causas evitáveis, Belo Horizonte, 1984-1998. Revista de Saúde Pública, São Paulo, v. 39, n. 1, p. 67-74, 2005.

CAMARGO, R. M. S. et al. Consumo alimentar de gestantes atendidas em ambulatório de pré-natal da rede pública. Ciência, Cuidado e Saúde, Cuiabá, v. 11, n. 3, p. 489-496, 2012.
CERVATO-MANCUSO, A. M. et al. A atuação do nutricionista na Atenção Básica à Saúde em um grande centro urbano. Ciência e Saúde Coletiva, Rio de Janeiro, v. 17, n. 12, p. 3.289-3.300, 2012.

GEUS, L. M. M. et al. A importância na inserção do nutricionista na Estratégia Saúde da Família. Ciência e Saúde Coleti$v a$, Rio de Janeiro, v. 16, supl. 1, p. 797-804, 2011.

LIBERA, B. D. et al. Avaliação da assistência pré-natal na perspectiva de puérperas e profissionais de saúde. Ciência e Saúde Coletiva, Rio de Janeiro, v. 16, n. 12, p. 4.855-4.864, dec. 2011.

MELERE, C. et al. Índice de alimentação saudável para gestantes: adaptação para uso em gestantes brasileiras. Rev. Saúde Pública, São Paulo, v. 47, n. 1, p. 20-28, feb. 2013.

PÁDUA, J. G.; BOOG, M. C. F. Avaliação da inserção do nutricionista na Rede Básica de Saúde dos municípios da Região Metropolitana de Campinas. Revista de Nutrição, Campinas, v. 19, n. 4, p. 413-424, aug. 2006.

RAMALHO, R. A. et al. Associação entre a deficiência de vitamina A e a situação sociodemográfica das mães e recém-nascidos. Revista da Associação Médica Brasileira, Rio de Janeiro, v. 52, n. 3, p. 170-175, 2006.

RIBAS, D. L. B. et al. Saúde e estado nutricional infantil de uma população da região Centro-Oeste do Brasil. Revista de Saúde Pública, São Paulo, v. 33, n. 4, p. 358-65, 1999.

RISSIN, A. et al. Retardo estatural em menores de cinco anos: um estudo "baseline". Ciência \& Saúde Coletiva, Rio de Janeiro, v. 16, n. 10, p. 4.067-4.076, 2011.

ROCHA, B. S.; ANDRADE, M. A promoção da saúde na assistência pré-natal realizada pelos enfermeiros no programa de saúde da família. Informe-se em Promoção da Saúde, v. 4, n. 2. p. 28-30, 2008.

SILVA, L. S. V. et al. Micronutrientes na gestação e lactação. Revista Brasileira de Saúde Materno Infantil, Recife, v. 7, n. 3, p. 237-244, set. 2007.

VARGAS, G. S. et al. Atuação dos profissionais de saúde da estratégia saúde da família: promoção da prática do aleitamento materno. Revista Baiana de Enfermagem, Salvador, v. 30, n. 2, p. 1-9, abr./jun. 2016.

VIEIRA, V. L.; SOUZA, J. M. P.; CERVATO-MANCUSO, A. M. Insegurança alimentar, vínculo mãe-filho e desnutrição infantil em área de alta vulnerabilidade social. Revista Brasileira de Saúde Materno Infantil, São Paulo, v. 10, n. 2, p. 199-207, 2010.

VÍTOLO, M. R. Nutrição: da gestação ao envelhecimento. Rio de Janeiro: Rubio, 2015. 